\title{
Prospects for Gamma-Ray Burst detection by the Cherenkov Telescope Array
}

\author{
E. Bissaldi ${ }^{1,2, a}$, T. Di Girolamo ${ }^{3,4}$, F. Di Pierro ${ }^{5}$, T. Gasparetto ${ }^{6}$, F. Longo ${ }^{6,7}$, P. Vallania ${ }^{5,8}$, and \\ C. Vigorito ${ }^{8,9}$ for the CTA Consortium ${ }^{10}$ \\ ${ }^{1}$ Politecnico di Bari, Bari, Italy \\ ${ }^{2}$ INFN - Sezione di Bari, Bari, Italy \\ ${ }^{3}$ Università degli Studi di Napoli "Federico II", Napoli, Italy \\ ${ }^{4}$ INFN - Sezione di Napoli, Napoli, Italy \\ ${ }^{5}$ INAF - OAT, Torino, Italy \\ ${ }^{6}$ Università degli Studi di Trieste, Trieste, Italy \\ ${ }^{7}$ INFN - Sezione di Trieste-Udine, Trieste, Italy \\ ${ }^{8}$ INFN - Sezione di Torino, Torino, Italy \\ ${ }^{9}$ Università degli Studi di Torino, Torino, Italy \\ ${ }^{10}$ See www.cta-observatory . org for full author \& affiliation list
}

\begin{abstract}
The Large Area Telescope (LAT) on the Fermi satellite is expected to publish a catalogue with more than 100 Gamma-Ray Bursts (GRBs) detected above $100 \mathrm{MeV}$ thanks to a new detection algorithm and a new event reconstruction. This work aims at revising the prospects for GRB alerts with the Cherenkov Telescope Array (CTA) based on the new LAT results. We start considering the simulation of the observations with the full CTA of two extremely bright events, the long GRB 130427A and the short GRB 090510, then we investigate how these GRBs would be observed by a particular configuration of the array with the telescopes pointing to different directions in what is called the "coupled divergent mode".
\end{abstract}

\section{The CTA observatory}

The Cherenkov Telescope Array (CTA) is a worldwide project aiming at building and operating the next generation of Imaging Atmospheric Cherenkov Telescopes (IACTs). In its baseline design, two huge arrays are foreseen, one in the Southern (Armazones, Chile) and one in the Northern (La Palma, Spain) hemisphere. In its current design, more than 100 telescopes will extend the energy range of currently operating IACTs especially at higher energies and will improve the sensitivity by about one order of magnitude with better angular and energy resolutions. In order to achieve these goals, a combination of different types of telescopes is necessary. Large Size Telescopes (LSTs), with a diameter of $23 \mathrm{~m}$, will observe the energy region between $\sim 20$ and $\sim 200 \mathrm{GeV}$, having a compact placement in both Northern and Southern sites. Near the low-energy threshold, the number of source photons is relatively high but the Cherenkov image is poor, therefore a few huge telescopes are used to collect

a speaker, e-mail: elisabetta.bissaldi@ba.infn.it 
faint showers. Medium Size Telescopes (MSTs), with a diameter of $12 \mathrm{~m}$, will be sensitive in the energy range between $\sim 100 \mathrm{GeV}$ and $\sim 10 \mathrm{TeV}$, arranged on about a $\mathrm{km}^{2}$ area. At these energies, the Cherenkov signal starts to increase while the source flux rapidly fades, so an intermediate choice for number and dimensions of the telescopes is optimal. Small Size Telescopes (SSTs), with a diameter of $4 \mathrm{~m}$, will operate in the energy range between $\sim 5$ and $\sim 300 \mathrm{TeV}$, and will cover a $3-4 \mathrm{~km}^{2}$ area. The current CTA baseline array layout consists of 4 LSTs and 25 MSTs in the Southern Site and 4 LSTs and 15 MSTs in the Northern Site. 70 SSTs will be built only in the Southern site, where most of the Galactic plane is visible.

\section{High-energy GRB observations}

Gamma-Ray Bursts (GRBs) represent a very interesting case study in astrophysics, mainly due to their multi-disciplinary nature. At present time, a GRB can trigger one or more of the dedicated instruments based on several satellites orbiting around the Earth, such as Swift, Fermi, MAXI or INTEGRAL. The observed keV-MeV prompt emission may be accompanied by an X-ray, optical or radio afterglow. Rapid follow-up of the prompt keV-MeV emission is possible thanks to communication through the Gamma-ray Coordinates Network $(\mathrm{GCN})$, where the GRB position is spread out in real time to all other observatories. This includes all currently operative IACTs like MAGIC, H.E.S.S., and VERITAS. Unfortunately, none of them ever succeeded in capturing a high-energy signal from a GRB, but several upper limits from a single or from a sample of bursts were published by each collaboration over the last years.

Current prospects for very high-energy GRB observations by CTA $[1,2]$ are based on extrapolations taken either from the GRB spectral parameters published in the catalogs of the Burst and Transient Source Experiment (BATSE, $20 \mathrm{keV-2} \mathrm{MeV)} \mathrm{and} \mathrm{of} \mathrm{the} \mathrm{Swift} \mathrm{Burst} \mathrm{Alert} \mathrm{Telescope} \mathrm{(BAT,}$ $15-150 \mathrm{keV}$ ), or from some very energetic GRBs detected by the Fermi instruments before 2012.

Our aim is to expand these samples with the newest results obtained by both instruments on-board Fermi, namely the Gamma-Ray Burst Monitor (GBM, $8 \mathrm{keV}-40 \mathrm{MeV}$ ) and the Large Area Telescope (LAT, $\sim 30 \mathrm{MeV}-300 \mathrm{GeV}$ ). We are currently preparing a library of GRBs observed at different posttrigger epochs, which includes $\sim 1000$ GRBs from the second GBM spectral catalog [3], 35 GRBs from the first LAT GRB catalog [4], and 130 new GRBs from the second LAT GRB catalog (in preparation, [5]). A subsample of these GRBs also comes with redshift information.

For the highest energy bursts, we extrapolate the flux to CTA energies by assuming that the TeV flux follows the time evolution of the $\mathrm{GeV}$ flux. Here we present the analysis results for two particular bursts detected by LAT, namely the long GRB 130427A and the short GRB 090510.

\section{Simulation of GRB observations}

To estimate the detectability of a burst, we make use of the ctools, a software package specifically developed for the scientific analysis of CTA data (v0.9.0) ${ }^{1}$.

Our first test case is GRB 130427A, which represents the current record holder of the highest energy photon ever observed from a GRB $(95 \mathrm{GeV})$. Moreover, it was detected at a low redshift of 0.34 and therefore followed-up by a very large number of telescopes. Observations by VERITAS began almost 20 hours post trigger and led to an upper limit (UL) of $3.3 \times 10^{-12} \mathrm{erg} \mathrm{cm}^{-2} \mathrm{~s}^{-1}$. This UL was used to cross-check our extrapolation to the CTA energies of the spectrum measured by LAT, which is described by a spectral index $\gamma=-2.2$ [6], almost constant from $400 \mathrm{~s}$ up to $70 \mathrm{ks}$ post trigger,

\footnotetext{
${ }^{1}$ http://cta.irap.omp.eu/ctools/index.html
} 

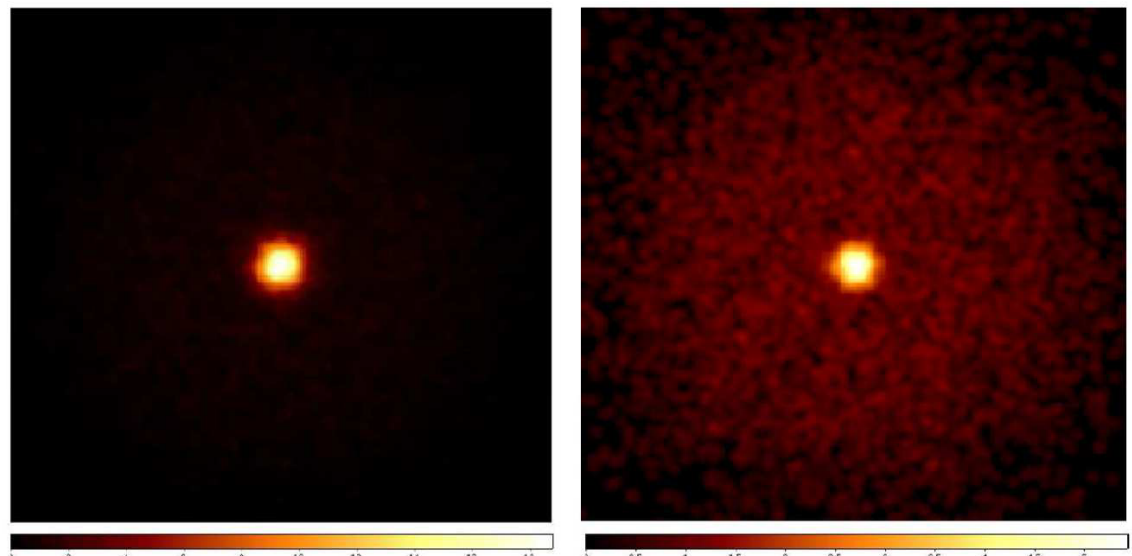

Figure 1. Simulations of GRB 130427 A with ctools. The camera images are divided into $200 \times 200$ bins of $0.02^{\circ}$. The color scale gives the counts/bin after Gaussian smoothing. Left panel: Count map as seen by CTA North in 10 min starting from 1 ks after the trigger. Right panel: Count map as seen by CTA North in 30 min starting from $10 \mathrm{ks}$ after the trigger.
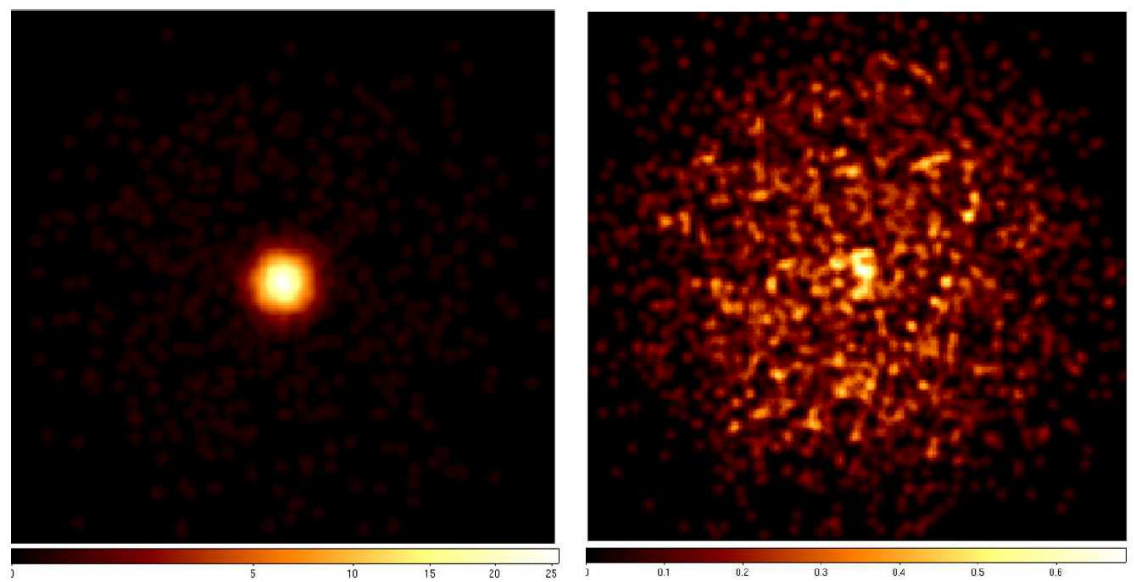

Figure 2. Simulations of GRB 090510 with ctools. Binning and color code as in Fig. 1. Left panel: Count map as seen by CTA North in $100 \mathrm{~s}$ starting from $100 \mathrm{~s}$ after the trigger. Right panel: Count map as seen by CTA North in $500 \mathrm{~s}$ starting from $1 \mathrm{ks}$ after the trigger.

and by a power-law decay with a temporal index $\tau=-1.35$ (valid for $\mathrm{t}>380 \mathrm{~s}$ ). For each GRB, an intrinsic spectrum of $(d N / d E) \propto E^{\gamma}$ is assumed.

The second test case is the short burst GRB 090510, also detected by LAT and located at a relatively low redshift of 0.9 . In this case, the highest-energy photon detected is a $31 \mathrm{GeV}$ one. The spectral parameters adopted in the simulation (taken from [7]) are $\gamma=-1.6$ for $t<200 \mathrm{~s}$ and $\gamma=-2.5$ at later times, while the temporal parameter (taken from [8]) is $\tau=-1.38$.

We assume that the bursts are observed on axis with respect to the CTA array and at a zenith angle $\theta=20^{\circ}$, a value provided in the latest CTA large-scale Monte Carlo simulations (Prod-2) [9]. 
In each case, we simulated two possible observations by CTA, namely (A) a small time-window at early post-trigger times, covering the end of the GRB prompt emission period, and (B) a large timewindow at late post-trigger times, covering the GRB afterglow emission period. For GRB 130427A, we simulated observation (A) lasting $10 \mathrm{~min}$ at $\mathrm{t}=1 \mathrm{ks}$ post trigger and observation (B) lasting half an hour at $t=10 \mathrm{ks}$ post trigger. For GRB 090510, observation (A) starts at $t=100 \mathrm{~s}$ post trigger and lasts $100 \mathrm{~s}$ while observation (B) starts at $\mathrm{t}=1 \mathrm{ks}$ post trigger and lasts $500 \mathrm{~s}$. In the case of GRB 130427A, we also included the effect of the EBL absorption, using the EBL model of [10]. For simplicity, in our simulations we extended the spectrum only up to $1 \mathrm{TeV}$, the maximum energy after which the source is assumed to be totally absorbed. Therefore, the energy range used in our GRB 130427A simulations is $50 \mathrm{GeV}-1 \mathrm{TeV}$. In the case of GRB 090510 we neglected the effect of EBL absorption and limited the energy range to $50-100 \mathrm{GeV}$, since the burst occured at a higher redshift than GRB 130427A.

The count maps of the simulated observations of GRB 130427A and GRB 090510 are displayed in Figures 1 and 2, respectively. In each case, left panels refer to observation (A) and right panels to observation (B). Count maps were obtained using the ctools functions ctobssim and ctbin and adopting the official CTA instrument response functions (IRFs) ${ }^{2}$. We made use of the North_0.5h IRF for observation (A) and of the North_5h IRF for observation (B). The decision of using a more conservative IRF for longer observations was driven by the need of reducing the increased background contribution.

A preliminary ctlike analysis was performed on all simulated observations, getting a significant detection in cases (A) and (B) of GRB 130427A and in case (A) of GRB 090510. Further analysis of other bright Fermi GRBs with known redshift is in progress.

In the future, we plan to use different pointing configurations of the array and to study the performance of the "coupled divergent mode" for the CTA observatory, in which pairs of telescopes are pointing to slightly different positions on the sky. Moreover, we intend to explore deeper the contribution of the MSTs in GRB searches.

\section{Acknowledgments}

We gratefully acknowledge support from the agencies and organizations listed under Funding Agencies at this website: http://www.cta-observatory.org/. E.B. is supported by the Italian Fondo di Sviluppo e Coesione 2007-2013 - APQ Ricerca Regione Puglia “Future In Research".

\section{References}

[1] S. Inoue et al., Astroparticle Physics 43252 (2012)

[2] R. Gilmore et al., Experimental Astronomy 35413 (2013)

[3] D. Gruber et al., The Astrophysical Journal Supplement Series 21112 (2014)

[4] M. Ackermann et al., The Astrophysical Journal Supplement Series 20911 (2013)

[5] G. Vianello et al., AAS Meeting Proceedings 227 id. 416.01 (2016)

[6] M. Ackermann et al., Science 34342 (2014)

[7] M. Ackermann et al., The Astrophysical Journal 7161178 (2010)

[8] M. De Pasquale et al., The Astrophysical Journal Letters 709146 (2010)

[9] T. Hassan et al., Proceedings of Science (ICRC2015) 971 (2015)

[10] A. Franceschini et al., Astronomy \& Astrophysics 487837 (2008)

\footnotetext{
${ }^{2}$ https://portal.cta-observatory.org/Pages/CTA-Performance.aspx
} 